\title{
News, Tipps, Termine
}

Thomas Vogt und David Vogel

\section{Ausgezeichet}

Florian Frick (Cornell University) wurde für seine Dissertation Combinatorial Restrictions on Cell Complexes mit dem Richard-Rado-Preis 2016 der DMV-Fachgruppe Diskrete Mathematik ausgezeichnet. Der Preis wird alle zwei Jahre auf dem Symposium Diskrete Mathematik der Fachgruppe für eine Promotion aus den vergangenen zwei Jahren verliehen und ist mit 1000 Euro, gestiftet vom SpringerVerlag Heidelberg, dotiert. Ein einzelner Juror aus dem Ausland trifft jeweils die Entscheidung: Diesmal hatte Ben Green (Oxford) aus zehn erstklassigen Promotionen auszuwählen.

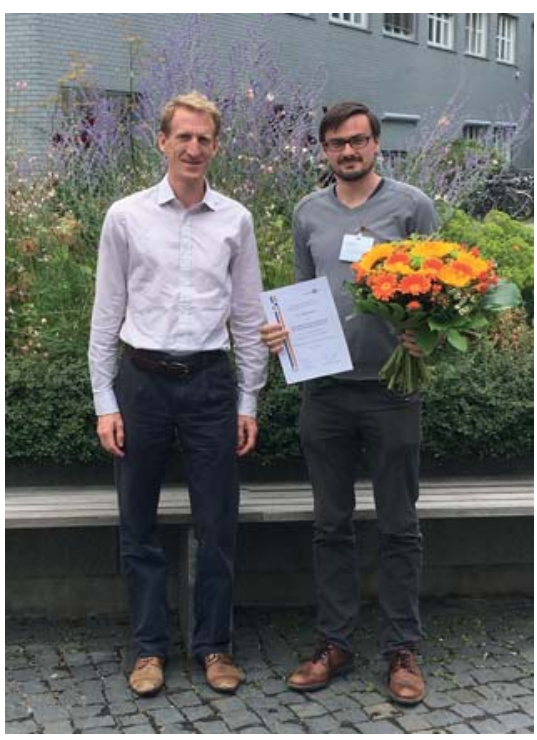

Ben Green und Florian Frick

(Foto: Christoph Kusch)

\section{Gesiebt}

Eine Newcomerin mit 938376I Dezimalstellen erobert Platz sieben (Primplatz vier) in Chris Caldwells Datenbank der größten bekannten Primzahlen. Szabolcs Peter aus Ungarn fand die Primzahl, indem er dem Crowd Computing Projekt PrimeGrid private Rechenkapazität stellte. Am 31. Oktober 2016 schloss Peters Computer eine fast neuntägige Berechnung ab, die zeigte, dass der Ausdruck $10223 \cdot\left(2^{31172165}\right)+1$ prim ist. Ausgeschlossen ist damit auch, dass es sich beim Koeffizienten der Zweierpotenz um eine sogenannte Sierpiński-Zahl handelt, denn dafür dürfte der Ausdruck mit keinem Exponenten je prim sein. Vor Peters Fund wurde 10223 als eine Kandidatin für die kleinste Sierpiński-Zahl gehandelt. Minumumstitel-Verteidigerin ist 78557 - dank Peter und PrimeGrid. Sucht man große Primzahlen, sind SierpińksiZahlen von eher geringer Bedeutung. Zwar kann man im
Prinzip gleich die komplette, unendliche Exponentialfolge aussieben, erhält damit aber ein allzu luftiges Sieb mit exponentiell ausweitenden Maschen.

Die Zahl im PDF: http://www2.epcc.ed.ac.uk/ ibethune/ files/SBI2.pdf (Ausdruck nicht empfohlen)

\section{Umstritten}

Schon ab I. I.2016 soll er trotz aller Proteste gelten: Der umstrittene neue Rahmenvertrag über Intranetnutzungen an öffentlichen Hochschulen gemäß $§ 52 a$ UrhG regelt nunmehr die Einzelnutzung von Schriftwerken und Auszügen zum Zweck der Lehre und Forschung, beispielsweise in digitalen Semesterapparaten. Tritt eine Hochschule dem Vertrag zwischen VG Wort und der KMK vom Oktober 2016 bei, müsste sie 0,008 Euro pro Seite und Unterrichtsteilnehmer zahlen. Die damit abgeschaffte Pauschalabrechnung wäre den meisten Hochschulen jedoch nach wie vor lieber. Sie befürchten, dass die neue Regelung unverhältnismäßigen Aufwand schaffen könnte. In der Hoffnung auf Neuverhandlungen empfiehlt das Aktionsbündnis „Urheberecht für Bildung und Wissenschaft" in einer Pressemeldung vom 23. II. 2016, dem Vertrag nicht beizutreten.

www.urheberrechtsbuendnis.de/pressemitteilung04I6. html.de

\section{Ausgeschrieben}

Der Ars legendi-Fakultätenpreis für exzellente Lehre in Mathematik und Naturwissenschaften ist für das Jahr 2017 ausgeschrieben. Bitte bewerben Sie sich bis zum 6. Januar 2017!

Der Stifterverband für die Deutsche Wissenschaft, die Deutsche Mathematiker-Vereinigung, die Deutsche Physikalische Gesellschaft, die Gesellschaft Deutscher Chemiker und der Verband Biologie, Biowissenschaften und Biomedizin in Deutschland loben den Preis zum vierten Mal aus. Er wird jährlich in den Kategorien Biowissenschaften, Chemie, Mathematik und Physik verliehen. Der Preis ist mit jeweils 5000 Euro dotiert.

Alle weiteren Informationen zu Preis und Bewerbung finden Sie auf www.dmv.mathematik.de.

\section{Gefördert I}

(DFG) Moderne Hochleistungscomputer mit mathematischer Software haben gerade im Bereich der Algebra, wo exakte Berechnungen unumgänglich sind, ein enormes Rechenpotenzial, das bisher nur unzureichend genutzt wird. Die Wissenschaftlerinnen und Wissenschaft- 
ler des Sonderforschungsbereichs/Transregio „Symbolische Werkzeuge in der Mathematik und ihre Anwendung" planen, bestehende, zu großen Teilen von ihnen selbst entwickelte Computeralgebrasysteme, weiterzuentwickeln. Damit sollen einerseits grundlagenwissenschaftliche mathematische Fragestellungen beantwortet werden; andererseits soll die Software als Open-SourceSystem allgemein verfügbar gemacht werden, wie die DFG mitteilte (http://tinyurl.com/hcs3zxp).

Sprecherhochschule: Technische Universität Kaiserslautern, Sprecher: Professor Dr. Gunter Malle; weitere antragstellende Hochschulen: Rheinisch-Westfälische Technische Hochschule Aachen, Universität des Saarlandes.

\section{Gefördert II}

(DFG) Die symplektische Geometrie hat ihre Wurzeln in der klassischen Mechanik. Sie erlaubt dort eine koordinatenfreie Formulierung der zentralen Bewegungsgleichungen und damit ein tieferes Verständnis der zugrunde liegenden Dynamik. Der Sonderforschungsbereich/Transregio „Symplektische Strukturen in Geometrie, Algebra und Dynamik" untersucht symplektische Strukturen und die Anwendungen symplektischer Techniken auf Themenstellungen aus den Bereichen Geometrie, Algebra, Dynamische Systeme, Topologie, Kombinatorik und Optimierung. Der Verbund knüpft dabei Beziehungen zu Disziplinen, in denen das Potenzial eines symplektischen Zugangs bislang kaum oder noch nicht voll realisiert ist, oder die ihrerseits neue Methodologien zum Studium symplektischer Fragen beitragen können, beispielsweise die Informatik, wie die DFG mitteilte (http://tinyurl.com/hcs3zxp).

Sprecherhochschule: Universität zu Köln, Sprecher: Professor Dr. Hansjörg Geiges; weitere antragstellende Hochschule: Ruhr-Universität Bochum.

\section{Aufgerufen}

Ab sofort - und noch bis 14. Februar 2017 - ist der (natur-)wissenschaftliche Nachwuchs aller Phasen (undergrad, PhD or postdoc) aufgerufen, sich für das 5 . Heidelberg Laureate Forum (HLF) zu bewerben. Am bequemsten geht das online über dieses Formular:

http://application.heidelberg-laureate-forum.org

Das HLF findet vom 24.-29. September 2017 in Heidelberg statt. Ebenfalls ab sofort können sich Graduierte in den Naturwissenschaften wieder für den Klaus Tschira Preis für verständliche Wissenschaft bewerben, der in den Bereichen Biologie, Informatik, Mathematik, Neurowissenschaften und Physik ausgelobt wird. Einsendeschluss für die Textbeiträge ist der 28. Februar 2017. Der Klaus Tschira Preis ist mit jeweils 5000 Euro dotiert. Außerdem werden die Beiträge unverändert als Sonderbeilage bei der ZEIT veröffentlicht.

\section{GHzürich}

\section{Professur für Mathematik}

\author{
$\rightarrow$ Im Departement Mathematik \\ (www.math.ethz.ch) der ETH Zürich \\ ist die oben erwähnte Position zu \\ besetzen.
}

$\rightarrow$ Kandidatinnen und Kandidaten leisten weltweit herausragende Forschung in einem Gebiet der Mathematik. Zusammen mit den Kolleginnen und Kollegen trägt die neue Professorin oder der neue Professor die Verantwortung für den Unterricht in Mathematik auf Stufe Bachelor (Deutsch oder Englisch), sowie auf Stufe Master (Englisch) für Studierende der Mathematik, der Naturund der Ingenieurwissenschaften.

Die Bereitschaft zur Zusammenarbeit innerhalb und ausserhalb der

ETH Zürich wird vorausgesetzt.

$\rightarrow$ Bitte bewerben Sie sich online über: www.facultyaffairs.ethz.ch

\section{$\rightarrow$ Bewerbungen mit Lebenslauf} und Publikationsliste, einer Beschreibung der beabsichtigten Forschungs- und Lehrtätigkeit sowie einer Beschreibung der drei bedeutendsten Leistungen sind bis zum 28. Februar 2017 einzureichen. Das Anschreiben ist an den Präsidenten der ETH Zürich, Prof. Dr. Lino Guzzella, zu richten. Die ETH Zürich setzt sich für Chancengleichheit, die Bedürfnisse von Dual Career Paaren und die Vereinbarkeit von Beruf und Familie ein. Wissenschaftlerinnen werden ausdrücklich zur Bewerbung aufgefordert. 


\section{Ausgegründet}

Stephanie Schiemann und ihr Kollege Robert Wöstenfeld, seit vielen Jahren für DMV-Schulprojekte und auch den Mathe-Adventskalender zuständig, haben mit Unterstützung der DMV die gemeinnützige $\mathrm{GmbH}$ Mathe im Leben, kurz MiL, gegründet. „Die gGmbH ermöglicht uns insbesondere, Spenden- und Sponsorengelder einzuwerben und davon Projekte und Personal zu finanzieren“, sagt der frisch gebackene Geschäftsführer Robert Wöstenfeld. In Kooperation mit der DMV realisiert MiL Mathe im Advent 2016, ein Projekt, bei dem sowohl einzelne Schüler und Schülerinnen sowie Schulklassen, als auch ganze Schulen oder interessierte Erwachsene die Mathematik mit Spaß und Kreativität entdecken können.

www.mathe-im-advent.de

\section{Termine}

19. Januar 2017, München

Gauß-Vorlesung: Mathematik an der Schnittstelle von Design und Technik, Prof. Dr. Helmut Pottmann

http://tinyurl.com/go2svpt

Helmut Pottmann ist Professor für Geometrie an der Technischen Universität Wien und wissenschaftlicher Leiter des Center for Geometry and Computational Design. Sein Arbeitsschwerpunkt ist geometrische Modellierung für industrielle Anwendungen. Er ist der Begründer der Architekturgeometrie. Viele der von Helmut Pottmann entwickelten Konzepte haben einen unmittelbaren praktischen Nutzen: wellenförmige Fassaden aus planaren Glasstücken, Mittagsschatten im Glashaus dank geschickt positionierter Stahlträger, selbsttragende Decken mit minimalem Materialverbrauch.

In seinem Vortrag wird Helmut Pottmann die Querbeziehungen zwischen mathematischer Theorie, der Erstellung von Computersimulation und der praktischen Umsetzung bei der Konstruktion von Gebäuden erläutern. Er wird zeigen, wie man durch gezielten Einsatz von Mathematik zu innovativeren Designs und effizienteren Herstellungsprozessen gelangt.

20. Januar 2017, Berlin

Mathekalender Preisverleihung

TU Berlin, AudiMax

14. Februar 2017, Heidelberg

Bewerbungsschluss fürs HLF

www.heidelberg-laureate-forum.org/

14. bis 18. Februar 2017, Stuttgart

Bildungsmesse didacta

www.messe-stuttgart.de/didacta/

27. Februar bis 3. März 2017, Potsdam

GDM Jahrestagung 2017 an der Universität Potsdam

Di. 28.2.2017: Lehrertag der GDM 2017, Kosten: 30 EUR

www.gdm-tagung.de/2017

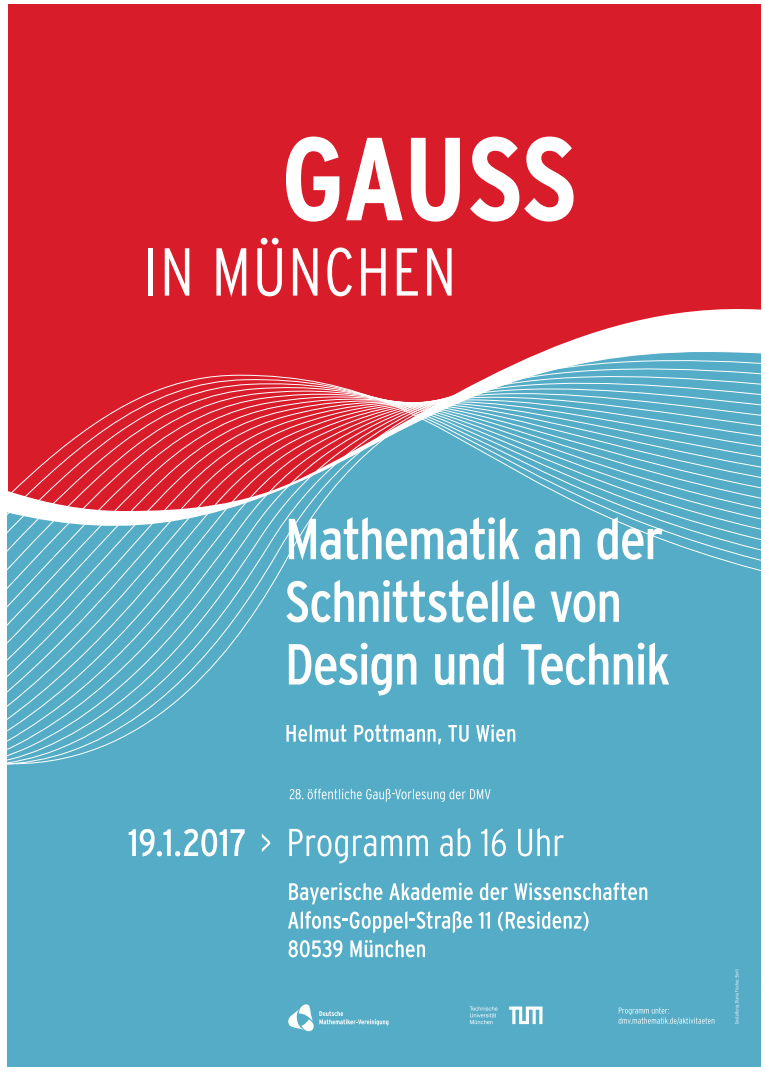

28. Februar 2017, Heidelberg

Bewerbungsschluss für den Klartext!-Preis der Klaus Tschira Stiftung www.klaus-tschira-preis.info

16. März 2017, bundesweit Kängurutag der Mathematik www.mathe-kaenguru.de

23. bis 25. März 2017, Darmstadt Forum für Begabtenförderung Mathematik an der Hochschule Darmstadt www.bfmathematik.de/20-forum-in-darmstadt/

6. bis 10. April 2017, Aachen 108. MNU-Bundeskongress http://bundeskongress-2017.mnu.de/

David Vogel und Thomas Vogt, Medienbüro Mathematik, Freie Universität Berlin, Institut für Mathematik, Arnimallee 7, 14195 Berlin

Tel. (030)838 75657. medienbuero@mathematik.de 